\title{
Research and Implementation on Microwave Power Online Measurement and Compensation of Automatic Test System
}

\author{
Liang Qin*, Zhen Wang, Yuhang Kang and Xianjun Shi \\ Naval Aeronautical and Astronautical University, Shandong 264001, China \\ ${ }^{*}$ Corresponding author
}

\begin{abstract}
Aiming at the problem of microwave cable power attenuation of automatic test system, this paper puts forward to a microwave power online measurement and compensation method and designs power measuring equipment. Before the test process, it is controlled by automatic test program, the measurement cable outputs signal power, returns the analog voltage, and then adjusts microwave signal source power, compensate the cable power attenuation. Finally, this method has been proved and implemented by combining the examples.
\end{abstract}

Keywords-component; automatic test system, power attenuation, online measurement

\section{INTRODUCTION}

In the design process of automatic test system, the problems that the microwave cable is long and the signal attenuation is serious often occur. Generally by choosing high performance waveguide or compensating in the source of signal, and then measuring and verifying it rigorously to ensure that the power of the cable output is accurate. But with the equipment delivery time grows, the power attenuation characteristic of microwave cable will shift inevitably, and the preventive means which is taken in the design process can't deal with the long-term changes, a calibration which is conducted each year can't completely ensure the accuracy in the using process.

In order to ensure the accuracy of the microwave excitation signal, literature [1] researches the microwave source of automatic test system, literature [2] and literature [3] researches the calibration of microwave power, literature [4] establishes attenuation automatic test system. Aimed at implementing online measurement compensation for microwave power, this paper proposes a online measurement compensation method for microwave power, designs the measurement device of power, and implements online measurement and compensation for microwave signal power.

\section{THE BASIS PRINCIPLE}

As shown in figure 1 , the main equipment of microwave signal online measurement and compensation method which is presented in this paper is power measurement device, power measurement device is placed near the measured object, every time during the initialization stage of test task, the cable terminal power value of microwave signal source is measured, and then use the embedded controller to calculate, return the measurement voltage. According to return voltage which is measure by multi-meter, the automatic test system adjusts the power of microwave signal source, compensates the cable power attenuation. Under the control of switching value, power measurement device switches the microwave signal which has been compensated to the output port.

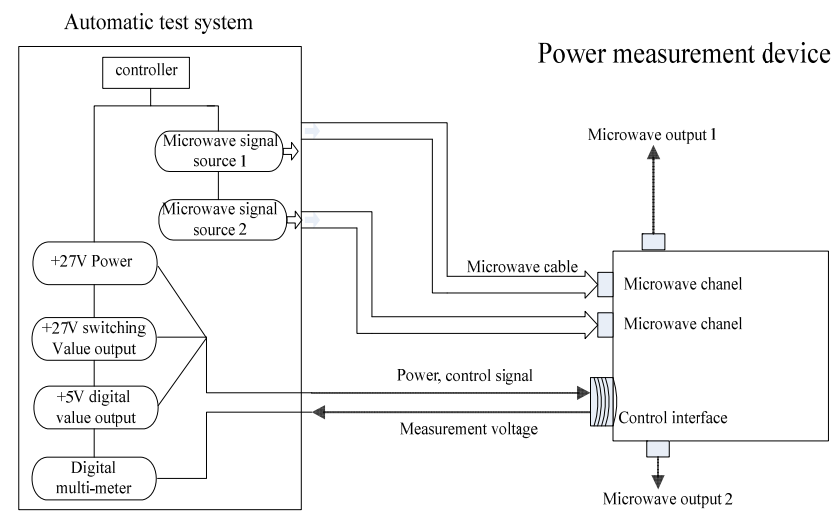

FIGURE I. ON-LINE MEASUREMENT AND COMPENSATION MODEL

The main functions of each part are as follows:

(1) $+27 \mathrm{~V}$ power: supply power for the P104 embedded computer, conditioning circuit and measurement circuit of power measurement device.

(2) $+27 \mathrm{~V}$ switching value: control the microwave switch of power measurement device, implement switch of several state such as channel 1 output, channel 1 measurement, channel 2 output, channel 2 measurement.

(3) $+5 \mathrm{~V}$ digital value: complete the communication between the automatic test system and the power measurement device, implement the function such as piecewise control and so on.

(4) Digital multi-meter: measure the feedback voltage of power measurement device

(5) Microwave signal source: supply microwave signal excitation for the object which is measured, the power measurement device which is designed in this paper supports 
the measurement and conversion for microwave signals from two channels

(6) Power measurement device: before the beginning of test process, switch the microwave signal to the measurement channel, the microwave power value is returned to the test device in the form of analog voltage. After the beginning of test process, switch the microwave signal to the output channel, and it is supplied for the object which is measured.

\section{Design Of Power Measurement DeVice}

The power measurement device is the core device of this method, the basis schematic is shown in Figure II.

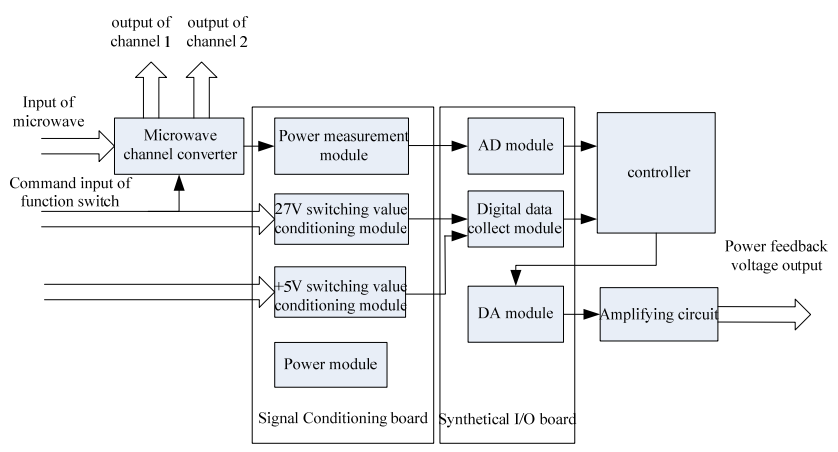

FIGURE II. SCHEMATIC OF POWER MEASUREMENT DEVICE

\section{A. Microwave Channel Converter}

Microwave channel converter is made up of microwave switch module and control command module, and it is mainly used to complete the conversion of microwave channel. Control command module is used to generate $+5 \mathrm{~V} /-5 \mathrm{~V}$ command to control the microwave switch module which make a conversion between channel 1 and channel 2 . the structure diagram of microwave channel converter is shown as figure III

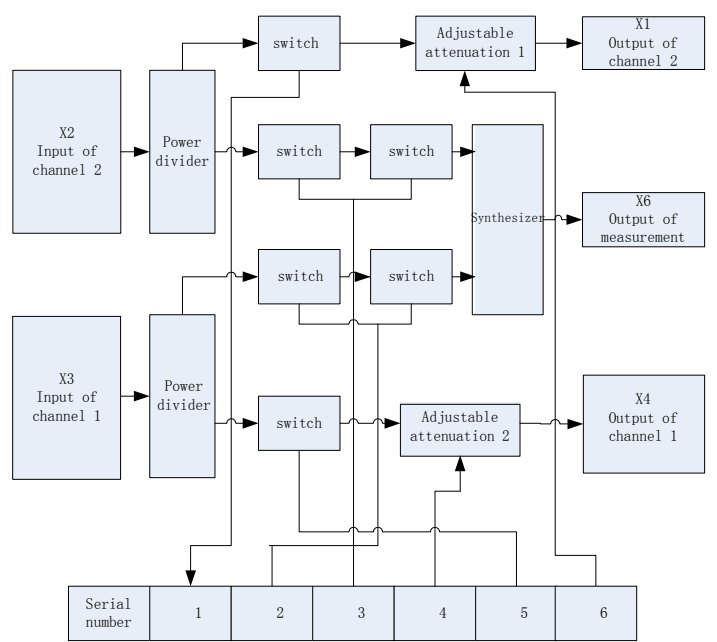

FIGURE III. STRUCTURE DIAGRAM OF MICROWAVE CHANNEL CONVERTER

The command control module is made up of relay group which forms the mutual lock. The upper computer controls the relay's action and outputs the switch command of microwave channel. The control command logic is shown in table 1.

TABLE I. CONTROL COMMAND LOGIC

\begin{tabular}{|c|c|c|c|c|c|c|}
\hline 1 & 2 & 3 & 4 & 5 & 6 & Gating state \\
\hline-5 & & & & & & $\mathrm{X} 2 \rightarrow \mathrm{X} 1$ turn on \\
\hline+5 & & & & & & $\mathrm{X} 2 \rightarrow \mathrm{X} 1$ turn off \\
\hline-5 & & & & & +5 & $\mathrm{X} 2 \rightarrow \mathrm{X} 1$ attenuation \\
\hline & & -5 & & & & $\mathrm{X} 2 \rightarrow \mathrm{X} 6$ turn on \\
\hline & & +5 & & & & $\mathrm{X} 2 \rightarrow \mathrm{X} 6$ turn off \\
\hline & & & & -5 & & $\mathrm{X} 3 \rightarrow \mathrm{X} 4$ turn on \\
\hline & & & & +5 & & $\mathrm{X} 3 \rightarrow \mathrm{X} 4$ turn off \\
\hline & & & +5 & -5 & & $\mathrm{X} 3 \rightarrow \mathrm{X} 4$ attenuation \\
\hline & -5 & & & & & $\mathrm{X} 3 \rightarrow \mathrm{X} 6$ turn on \\
\hline & +5 & & & & & $\mathrm{X} 3 \rightarrow \mathrm{X} 6$ turn off \\
\hline
\end{tabular}

B. Signal Conditioning Board

The signal conditioning board is made up of power module power measurement module and switching value control module, its main function is that complete the power conversion, measure the microwave power and control signal conditioning.

The power module need complete the conversion from $+27 \mathrm{~V}$ to $+5 \mathrm{~V}$ and $+15 \mathrm{~V}$, supplies the power to controller and circuit board. Power measurement module is mainly made up of the power measurement unit LT3533, the voltage signal which is generated by it is sent to AD module. The switching value conditioning module sends the switching value control signal which is $+27 \mathrm{~V}$ and $+5 \mathrm{~V}$ to the digital data collect module after conditioning.

\section{Synthetical I/O Board}

When the synthetical I/O board completes the collection of the voltage which is outputted by power measurement, $+27 \mathrm{~V}$ switching value and $+5 \mathrm{~V}$ switching value, these data will be transmitted to the controller by $\mathrm{PC} 104$ bus. After comprehensive justification of control program, determine the voltage value by controlling DA output.

The synthetical I/O board is mainly made up of PM518 which is based on PC104 bus, it has analog input and output functions, switching value input and output functions which is shown in figure IV.

\section{Controller}

The controller is mainly made up of Lan $\mathrm{Yu}$ science\&technology PCM-3386 embedded control main-board which is shown in figure 5. PCM-3386 is a embedded control module which has high cost-performance ratio and compact size. In family of PC104 specification board, it implements the powerful computation and interface function. PCM-3386 possesses embedded high performance 16 bit processor which is 32 bit RISC architecture and is compatible with the $80 \mathrm{C} 186$ processor, it has high performance, basic frequency reaches $100 \mathrm{MHz}$, built-in Ethernet reaches $100 \mathrm{M}$, and supports SDRAM of $1 \mathrm{MB}$, its command is compatible with other X86 microprocessors. 


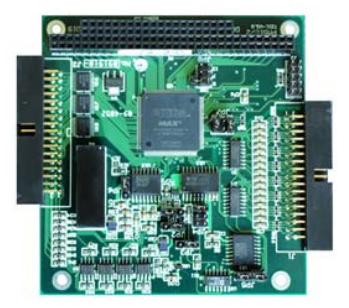

FIGURE IV. SYNTHETICAL I/O BOARD PM518

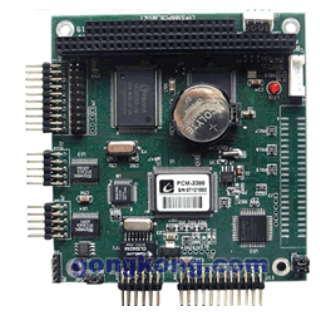

FIGURE V. EMBEDDED CONTROL MAIN-BOARD PCM-3386

\section{The Flow OF ONLINE MEASUREMENT COMPENSATION METHOD.}

Before the beginning of each test flow, starting with power measurement and compensation program at first. Automatic test system sends the command which switch microwave signal channels, control power measurement device transfer the signal to measurement channel. Send control command of $+27 \mathrm{~V}$ and $+5 \mathrm{~V}$ to control power measurement device select output formula. Set the initial power, adjust the value of step, return voltage standard value and output the signal. By measuring the power, power measurement device can get the voltage value, according with the formula, return voltage value can be achieved. Compare return voltage value and standard value by automatic test program, simultaneously, according with the step size to adjust the power of signal source. The cycle is 10 times at most before return voltage value reaching standard value. The concrete flow chart is shown in figure VI.

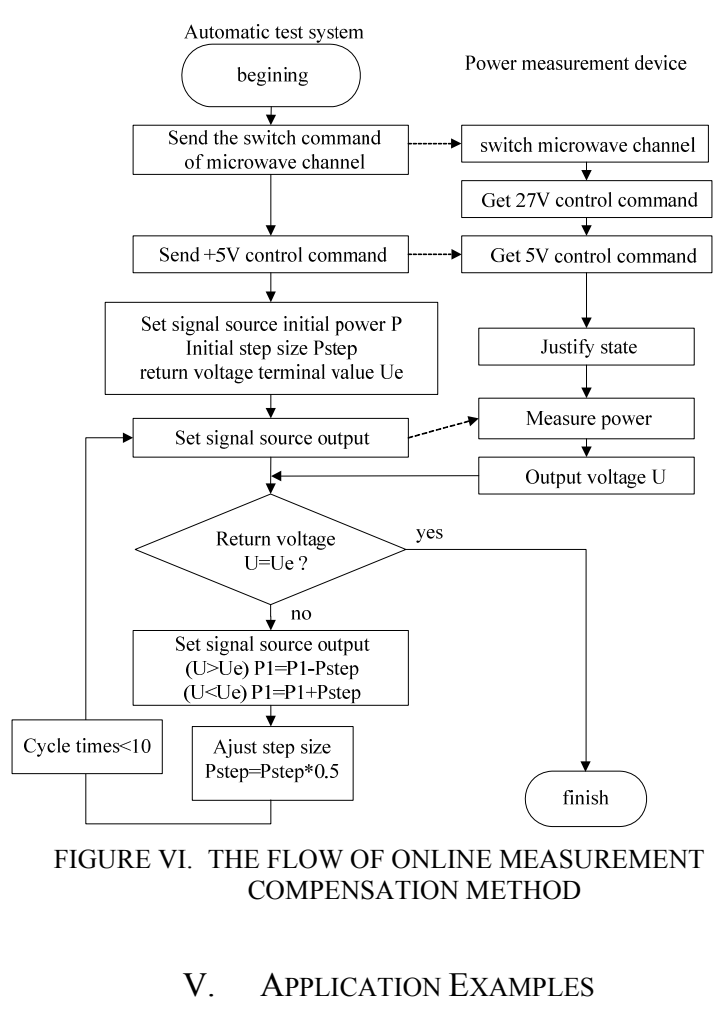

According to the design requirement, the output voltage of power measurement module needs to be transferred to the specified return voltage, this paper designs the output curve by using polynomial fitting method. Different output curves are needed under different command conditions, this paper designs control program, collects the digital value state comprehensively, judges the control state and generates the return voltage.

\section{A. The Design of Output Curve}

According to the requirement of test system design parameters and the static power test data of this device, the output curve data fitting program uses polynomial fitting method, gets the fitting formula and calculates the output voltage value. The specific flow chart is shown in figure VII. 


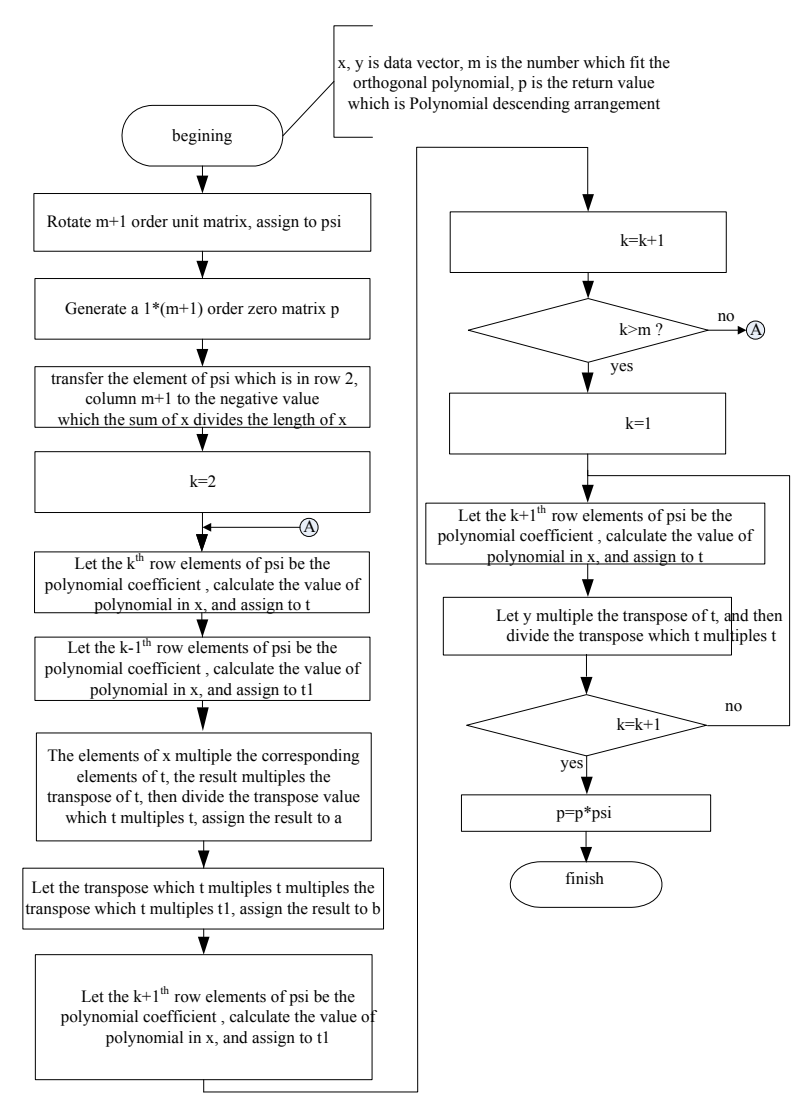

FIGURE VII. DATA FITTING FLOW CHART

Take channel 1 as an example, in the condition that the input power is between $-10 \mathrm{dBm}$ to $0 \mathrm{dBm}$, we can get the fitting formula:

$$
y=-1.3255 x^{3}+9.2377 x^{2}-0.0869 x-1.5
$$

$\mathrm{y}$ is the power measurement voltage output value of channel $1, \mathrm{x}$ is the voltage value which is measured by power measurement module. The formula is fitting result of threeorder polynomial, and the coefficient is the calculated by the fitting program. In the fitting process, the input vector is the voltage which is achieved by power measurement module, the output vector is design index requirement of test system.

\section{B. Design of Software}

The system software is made up of two parts which are respectively controller control program and data fitting program.

The controller control program deals with the result of digital input and power measurement synthetically, calls the data fitting program, outputs the power calibration voltage. The flow chart of controller control program is as shown in figure VIII.

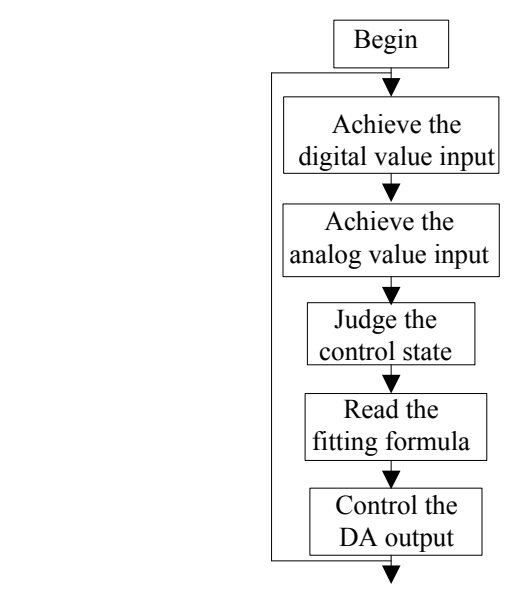

FIGURE VIII. FLOW CHART OF CONTROL PROGRAM

\section{CONCLUSION}

This paper proposes a microwave power on-line measurement compensation method of automatic test system, before each test task, measures the cable attenuation, adjusts the power of microwave signal source and compensates the power attenuation so that ensures the accuracy of excitation signal. The method overcomes the attenuation parameter drift during the verification period and achieves good effect in practical application, it can be used to the design of automatic test system.

\section{REFERENCE}

[1] [1] Zhou Ling, Xian Jingqian.The microwave source of automatic test system [J]. guidance and fuze, 1983,4(02):33-40.

[2] [2] Lou Hongying, Yang Zhong, Liu Fangjin. Automatic verification and calibration system of microwave broadband attenuation[J]. modern ladar, 2006,28(07):96-100.

[3] [3]Wang Yongzheng, Xiang Jin, Wang Bingwen. The development of microwave attenuation measurement technology[J]. Journal of Communication University of China, 2010, 17(01):1-11.

[4] [4]Wang Weibin, Qin Honglei. The implement of automatic test system for radio altimeter[J], Jounal of Electronic measurement and instrument, 2007,21(04):72-76.

[5] [5]Liu Jie, Liu Li. Automatic calibration of large attenuation based on Basic Visual 6[J]. Measurement technology,2009, 29(2):25-28. 